\title{
Clinical and Epidemiological Characteristics of Patients with Dientamoeba fragilis Infection
}

\author{
Lucía Miguel, ${ }^{1}$ Fernando Salvador, ${ }^{1 \star}$ Elena Sulleiro, ${ }^{2}$ Adrián Sánchez-Montalvá, ${ }^{1}$ \\ Daniel Molina-Morant, ${ }^{1}$ Isabel López, ${ }^{2}$ and Israel Molina ${ }^{1}$ \\ ${ }^{1}$ Department of Infectious Diseases, Vall d'Hebron University Hospital, Universitat Autónoma de Barcelona, PROSICS Barcelona, Barcelona, \\ Spain; ${ }^{2}$ Department of Microbiology, Vall d'Hebron University Hospital, PROSICS Barcelona, Barcelona, Spain
}

\begin{abstract}
Dientamoeba fragilis is an intestinal protozoan, usually considered nonpathogenic. However, in the last years, there has been an attempt to clarify its possible pathogenic role. We aim to evaluate the clinical and epidemiological characteristics of $D$. fragilis-infected patients. Adults with $D$. fragilis detection in feces who attended the Vall d'Hebron University Hospital (Barcelona, Spain) were evaluated retrospectively from April 2009 to March 2014. We classified the patients in asymptomatic, symptomatic without other causes except infection of $D$. fragilis, and symptomatic with another cause. Among symptomatic patients, treatment response was evaluated. One hundred eight patients were included. Sixty-three percent of the patients were immigrants, $29.6 \%$ were autochthonous, and $7.4 \%$ were travelers. Forty-nine (45.3\%) patients presented symptoms, and eosinophilia was observed in 26 (24.1\%) patients. Overall, 59 (54.7\%) patients were asymptomatic, 15 (13.8\%) presented symptoms which were attributable to other causes, and $34(31.5 \%)$ patients presented symptoms with no other causes. In this last group, 29 patients received specific treatment and $65.5 \%$ of them presented a complete resolution of the symptoms. The group of symptomatic patients with no other cause had more proportion of women, more proportion of autochthonous people, and were older compared with the group of asymptomatic patients. Dientamoeba fragilis infection should be considered as pathogenic when other causes are ruled out.
\end{abstract}

\section{INTRODUCTION}

Dientamoeba fragilis is an intestinal protozoan of worldwide distribution. ${ }^{1}$ Its pathogenic role and its life cycle are not well established, despite being observed for the first time in 1909 and described as a new species in 1918 by Jepps and Dobell. ${ }^{2}$ The main host is humans, but it was also detected in nonhuman primates, rodents, sheeps, and pigs. ${ }^{3}$

Although $D$. fragilis lacks flagella, it is classified as a flagellated protozoan as it shares ultrastructural morphological and antigenic similarities with protozoan of this family, such as Trichomonas spp. ${ }^{4}$ It moves through the pseudopods emission and it is of a small size $(5-15 \mu \mathrm{m}){ }^{5}$ It can be located in any section of the colon, although its preferable habitat is the cecum and the ascending colon. ${ }^{6}$ The life cycle is not known with certainty. The cystic and precystic forms were recently described, besides the trophozoites that were already known. ${ }^{3}$ The cyst role in the transmission of infection remains unknown. The mechanism of transmission of $D$. fragilis is controversial: the most accepted one is the fecal-oral transmission, although it is also postulated that it could be transferred by helminth that acts as vectors, such as Enterobius vermicularis (this could explain the higher prevalence in children). Transmission is also higher in adverse hygienic and overcrowding conditions. ${ }^{7,8}$

It is a relatively frequent infection around the world, with variable data of prevalence according to series: $0.4-42 \%{ }^{9}$ The infection can be chronic and persist for years. The pediatric age is the group with higher prevalence and the group with longer duration of symptoms. ${ }^{2}$ In some series, it is the most observed protozoan in feces, and in others, the second one (after Giardia sp. or Blastocystis hominis). ${ }^{9}$ Despite it, there are few studies that suggest its potential pathogenic role, which is not well established, in part due to the great variability of symptoms that it can cause. It is also one of the

* Address correspondence to Fernando Salvador, Infectious Diseases Department, Vall d'Hebron University Hospital, $\mathrm{P}^{\circ}$ Vall d'Hebron 119129, Barcelona 08035, Spain. E-mail: fmsalvad@vhebron.net few protozoans, as well as Isospora belli, that can cause eosinophilia. It was seen in a study that $50 \%$ of the cases in children and $10 \%$ in adults had eosinophilia. ${ }^{10}$ There is no clear evidence if treatment is indicated in all patients. Moreover, treatment options have not been compared in observational studies or clinical trials.

In this study, we describe the epidemiological and clinical data of patients with $D$. fragilis infection. We try to evaluate risk factors associated with the presence of symptoms, and we describe the clinical and microbiological follow-up after antiparasitic treatment.

\section{METHODS}

It is an observational retrospective study carried out at the Department of Infectious Diseases together with the Department of Microbiology of the Vall d'Hebron University Hospital, a tertiary hospital included in the International Health Program of Catalan Health Institute (PROSICS). Adult patients (18 years or older) in whom $D$. fragilis in feces was detected during the study period (from April 2009 to March 2014) were included. Patients were identified for the study through the microbiological registry of the Parasitological Laboratory. During the study period, patients were included only if the characteristic binucleate form of $D$. fragilis trophozoite was observed by microscopic examination of stool samples using the Blagg's concentration technique (merthiolateiodine-formaldehyde concentration). Both inpatients and outpatients were included in the study; patients were included independently of the reason of the stool examination (eosinophilia, gastrointestinal symptoms, screening in asymptomatic immigrants, etc.) and the number of stool samples investigated.

Epidemiological and clinical characteristics of the patients were collected, including gender, age, country of origin, immunosuppressant conditions, presence of symptoms (diarrhea, abdominal pain, vomits, flatulence, dyspepsia, abdominal distension, and itching), and presence of eosinophilia (defined as eosinophil cell count $\geq 500$ cells $/ \mathrm{mm}^{3}$ and/or $\geq 7 \%$ ). Taking 
into account the epidemiological information, patients were classified into three epidemiological risk groups: autochthonous (patients born in Spain), immigrants (patients born in other country and currently residing in Spain), and travelers (patients born in Spain with a recent travel outside the country). It was also collected the presence of other parasites in feces and/or the existence of other causes for the symptoms. After checking the clinical histories, three groups of patients were established according to the clinical situation: asymptomatic, symptomatic with another possible cause, and symptomatic without other known cause except for the $D$. fragilis infection. In the last group, the clinical and microbiological response was evaluated in those patients who received specific treatment.

Regarding the statistical analysis, categorical variables were presented as absolute numbers and proportions, and the quantitative variables were expressed through medians and ranges. To compare the distribution of categorical variables, the $\mathrm{X}^{2}$ test was used, and the Mann-Whitney $U$ test for the quantitative variables. The results were considered statistically significant if the two-tailed $P$ value was $<0.05$. For the statistical analysis, SPSS for Windows was used (19.0 Version; SPSS, Inc., Chicago, IL).

The Strengthening the Reporting of Observational Studies in Epidemiology statement guidelines were used to improve the quality of the study. The study protocol was approved by the Ethical Review Board of the Vall d'Hebron University Hospital (Barcelona, Spain). Procedures were performed in accordance with the ethical standards laid down in the Declaration of Helsinki as revised in 2013. Given the retrospective nature of the study and the anonymization of the information, the Ethical Review Board considered that obtaining the informed consent was not mandatory, although oral informed consent was tried to be obtained by telephone calls.

\section{RESULTS}

Overall, 108 patients were included in the study. From the 108 patients, 57 (52.8\%) were women, and the median age was 38 (18-85) years. Seventeen (15.7\%) patients presented some kind of immunosuppressive condition, from whom 14 had HIV infection, with a median of CD4 cell count of 461 (30-875) cells $/ \mathrm{mm}^{3}$, two patients had a hematologic malignancy, and another one was receiving treatment with a monoclonal antibody (anti-tumor necrosis factor) because of a rheumatoid arthritis.

Regarding the origin, $63 \%(68 / 108)$ of the patients were immigrants with a median time of residence in Spain of 36 (4-64) months. The most frequent country of origin was Bolivia (27.9\% of immigrants) and the second one Equatorial Guinea (7.3\%). Thirty percent $(32 / 108)$ of the patients were born in Spain, and $7.4 \%(8 / 108)$ were recent travelers. Table 1 describes clinical and epidemiological characteristics of patients.

Forty-nine $(45.3 \%)$ patients presented some gastrointestinal symptom, and the duration of the symptoms was over 1 month in 35 of them (70\%). Twenty-six (24.1\%) patients presented eosinophilia: five of these patients had digestive symptoms besides the eosinophilia, whereas the 21 patients left only had eosinophilia. Among patients with eosinophilia, the median eosinophil cell count was 1,150 cells $/ \mathrm{mm}^{3}$ $(800-2,700)$ and 10 patients had also a helminthic infection
TABLE 1

Epidemiological and clinical characteristics of patients with Dientamoeba fragilis infection

\begin{tabular}{lc}
\hline \multicolumn{1}{c}{ Characteristics } & Number of patients $(N=108)$ \\
\hline Age (years) & $38(18-85)$ \\
Gender (women) & $57(52.7 \%)$ \\
Immunosuppressant conditions & $17(15.7 \%)$ \\
Human immunodeficiency virus infection & $14(12.9 \%)$ \\
Hematologic malignancies & $2(1.7 \%)$ \\
Rheumatoid arthritis & $1(1 \%)$ \\
Epidemiological risk groups & $68(63 \%)$ \\
Immigrants & $32(29.6 \%)$ \\
Autochthonous & $8(7.4 \%)$ \\
Travelers & \\
Symptoms: & $37(34.3 \%)$ \\
Diarrhea & $21(19.4 \%)$ \\
Abdominal pain & $5(4.6 \%)$ \\
Nausea or vomits & $11(10.2 \%)$ \\
Flatulence & $9(8.3 \%)$ \\
Abdominal distension & $5(4.5 \%)$ \\
Itching & \\
\hline
\end{tabular}

that could be the cause of the eosinophilia; hence, 16 patients have $D$. fragilis infection as the only found cause of eosinophilia.

It was observed that 59 (54.7\%) patients were completely asymptomatic, including 21 patients with eosinophilia without digestive symptoms. Fifteen (13.8\%) patients presented symptoms attributed to other causes. From them, seven patients had other intestinal parasitic infection, being Giardia lamblia and Strongyloides stercoralis the most frequent ones. Table 2 shows other intestinal parasites detected in the study group. Five patients had gastrointestinal diseases (bacterial overgrowth syndrome, vellositary atrophy due to angiotensin II receptor blockers, atrophic gastritis, and two had an inflammatory bowel disease), one patient presented dermatological disease, and two patients had other causes of gastrointestinal symptoms (adverse event due to lopinavir and wasting syndrome, both in HIV-infected patients). Thirty-four $(31.5 \%)$ patients presented symptoms with no other cause except $D$. fragilis infection, attributing the symptoms to this parasite.

Among the 34 patients with symptoms without other cause different from $D$. fragilis, 29 patients $(85.3 \%)$ received a specific treatment of $D$. fragilis $(25$ patients received

TABLE 2

Other intestinal parasites detected in stool samples of the study population

\begin{tabular}{ll}
\hline \multicolumn{1}{c}{ Parasites } & Number of patients $(N=108)$ \\
\hline Pathogenic parasites & \\
Hookworm & $3(10 \%)$ \\
Giardia lamblia & $5(16.6 \%)$ \\
Hymenolepis nana & $2(6.6 \%)$ \\
Strongyloides stercoralis & $5(16.6 \%)$ \\
Taenia sp. & $1(3.3 \%)$ \\
Trichuris trichiura & $1(3.3 \%)$ \\
Enterobius vermicularis & $3(10 \%)$ \\
Schistosoma intercalatum & $1(3.3 \%)$ \\
Parasites of minor importance & \\
Blastocystis hominis & $5(16.6 \%)$ \\
Nonpathogenic parasites & $2(6.6 \%)$ \\
Entamoeba coli & $1(3.3 \%)$ \\
lodamoeba butschlii & $1(3.3 \%)$ \\
Endolimax nana & \\
\hline
\end{tabular}


metronidazole, three paromomycin, and one iodoquinol). Of them, $65.5 \%$ presented a complete resolution of the symptoms and $24.1 \%$ a partial resolution. The microbiological evolution was checked only in 14 patients, with negative detection of $D$. fragilis in stool samples in 12 of them (85.7\%). We did not have available data about the untreated group.

Symptomatic patients (without other demonstrated cause) had higher proportion of women, higher proportion of autochthonous population, and were older compared with asymptomatic patients $(P<0.01)$. Information is summarized in Table 3 .

\section{DISCUSSION}

We performed a retrospective study including 108 patients with $D$. fragilis infection. Most of them were immigrants, $24.1 \%$ of the study cohort had eosinophilia, and $45.3 \%$ of them had symptoms. In the group of patients with symptoms without cause other than $D$. fragilis infection who received treatment, $65.5 \%$ presented a complete resolution. Women, locals, and old people could be more likely to have symptoms when $D$. fragilis is detected.

Dientamoeba fragilis has been considered as part of the commensal gut flora; however, in the last years, there has been an attempt to clarify its possible pathogenicity. ${ }^{9} \mathrm{Al}-$ though only a few studies have tried to demonstrate it, with different and nonconclusive results, most of them have been performed in children. ${ }^{2}$ It has been demonstrated in murine models that inoculated mice with $D$. fragilis had a greater rate of weight loss and diarrhea compared with those in the control group. Histopathological changes have been observed in the colon of mice infected by $D$. fragilis, such as lymphoid hyperplasia, intestinal fibrosis, and acute peri-appendicitis, which might support the pathogenic role of $D$. fragilis. ${ }^{11}$

In our study, population from different origins is represented, being most of them immigrants. However, it is noticeable that although the immigrants are the most representative population, they are not the ones who presented more symptoms. This can be partly explained because many of the stool sample examinations performed in immigrants were performed as part of the screening of imported diseases. However, median time of residence in our country of the immigrants included in the study was high (more than 3 years); hence, the infection could have been acquired in our country. Women apparently are more susceptible to develop symptoms related to $D$. fragilis infection, without an established cause. It has also been observed that, contrary to studies performed in pediatric ages, in our population, patients with higher predisposition to have symptoms were older patients. ${ }^{12}$ In our cohort, immunosuppression does not seem to determine the development of symptoms, but the number of patients is not high enough to draw conclusions.

TABLE 3

Comparison of clinical and epidemiological characteristics between symptomatic and asymptomatic patients with Dientamoeba fragilis infection

\begin{tabular}{lccr}
\hline & Symptomatic $(N=34)$ & Asymptomatic $(N=59)$ & $P$ value \\
\hline Age, years & $46.5(28-85)$ & $33(20-53)$ & $<0.001$ \\
Gender, male & $9(26.47 \%)$ & $22(37.29 \%)$ & 0.011 \\
Foreign origin & $16(47.05 \%)$ & $36(61.01 \%)$ & $<0.001$ \\
Immunosuppression & $4(11.76 \%)$ & $10(16.95 \%)$ & 0.061 \\
\hline
\end{tabular}

From our cohort of $D$. fragilis-infected patients, more than a third $(38.9 \%)$ presented symptoms that could not be explained by any other cause. This indicates that most of the patients had another cause which explains the clinical symptoms or remained asymptomatic. Therefore, other etiologies have to be investigated individually. Nevertheless, symptoms can be attributed to $D$. fragilis infection in some patients. From them, most of the patients received a specific treatment (almost all with metronidazole, possibly because of the availability of this drug in our field) and presented a favorable clinical evolution. It is important to note that scarce information is available regarding the efficacy of the current approved drugs for treating $D$. fragilis infection. ${ }^{13,14}$

As it has been observed in other retrospective studies, our results reinforce the hypothesis that $D$. fragilis presents pathogenic capacity, which could be the cause of multiple digestive symptoms. ${ }^{15}$ Dientamoeba fragilis symptoms are similar to other intestinal parasites. Then, within the study of intestinal disorders, it should be necessary to include stool sample examinations for parasite detection.

On the other hand, it is noticeable that almost a quarter of our patients presented eosinophilia, whereas in other studies with adults, the presence of eosinophilia was around $10 \%$ (higher in children). ${ }^{8}$ Dientamoeba fragilis should be included in the differential diagnosis in the study of eosinophilia. However, in our study, we do not have follow-up data about eosinophilia; so, our results must be analyzed with caution.

The main limitation of our study is that it is based on retrospective data, through checking clinical histories. Each patient's follow-up was different, and there was no uniformity in regard to the follow-up period. Moreover, concomitant exclusion of other causes of symptoms and eosinophilia could have been not always appropriately studied. However, it gives relevant information in adult population, where the information is very scarce.

In summary, $D$. fragilis is an infection in front of which there is no clear consensus of action. In addition, symptoms are very variable and not specific. Our study suggests that therapy against $D$. fragilis may be effective in some patients when no other causes of the symptoms are detected. However, prospective studies are needed to unveil the uncertainties of this parasite.

Received May 23, 2018. Accepted for publication August 5, 2018.

Published online September 17, 2018.

Authors' addresses: Lucía Miguel, Fernando Salvador, Adrián SánchezMontalvá, Daniel Molina-Morant, and Israel Molina, Department of Infectious Diseases, Vall d'Hebron University Hospital, Universitat Autònoma de Barcelona, PROSICS Barcelona, Barcelona, Spain, E-mails: lucy_zgz86@hotmail.com, fmsalvad@vhebron.net, adsanche@vhebron. net, dani.molina7@gmail.com, and imolina@vhebron.net. Elena Sulleiro and Isabel López, Department of Microbiology, Vall d'Hebron University Hospital, PROSICS Barcelona, Barcelona, Spain, E-mails: esulleir@ vhebron.net and maibel56@gmail.com.

\section{REFERENCES}

1. Cacciò SM et al., 2016. Multilocus sequence typing of Dientamoeba fragilis identified a major clone with widespread geographical distribution. Int J Parasitol 46: 793-798.

2. Norberg A, Nord CE, Evengard $B, 2003$. Dientamoeba fragilis a protozoan infection which may cause severe bowel distress. Clin Microbiol Infect 9: 65-68.

3. Stark D, García LS, Barratt JL, Phillips O, Roberts T, Marriott D, Harkness J, Ellis JT, 2014. Description of Dientamoeba fragilis 
cyst and precystic forms from human samples. J Clin Microbiol 52: $2680-2683$.

4. Windsor JJ, Johnson EH, 1998. Dientamoeba fragilis: the unflagelated flagellate. Br J Biomed Sci 55: 172-175.

5. Johnson EH, Windsor JJ, Clark C, 2004. Emerging from obscurity: biological, clinical, and diagnostic aspects of Dientamoeba fragilis. Clin Microbiol Rev 17: 553-570.

6. Stark D, Barratt J, Chan D, Ellis JT, 2016. Dientamoeba fragilis, the neglected trichomonad of the human bowel. Clin Microbiol Rev 29: 553-580.

7. Ockert G, Schmidt T, 1976. Evidence of Dientamoeba fragilis in Enterobius eggs using isoelectric point determination. $\mathrm{J} \mathrm{Hyg}$ Epidemiol Microbiol Immunol 20: 76-81.

8. Boga JA, Rojo S, Fernández J, Rodríguez M, Iglesias C, MartínezCamblor P, Vázquez F, Rodríguez-Guardado A, 2016. Is the treatment of Enterobius vermicularis co-infection necessary to eradicate Dientamoeba fragilis infection? Int J Infect Dis 49: 59-61.

9. Stark D, Barratt J, Roberts T, Marriott D, Harkness J, Ellis J, 2010. A review of the clinical presentation of dientamoebiasis. Am J Trop Med Hyg 82: 614-619.
10. Stark D, Beebe N, Marriott D, Ellis J, Harkness J, 2005. Prospective study of the prevalence, genotyping, and clinical relevance of Dientamoeba fragilis infections in an Australian population. J Clin Microbiol 43: 2718-2723.

11. Munasinghe VS, Vella GN, Ellis JT, Windsor PA, Stark D, 2013. Cyst formation and faecal-oral transmission of Dientamoeba fragilis-the missing link in the life cycle of an emerging pathogen. Int J Parasitol 43: 879-883.

12. Holtman GA, Kranenberg JJ, Blanker MH, Ott A, Lisman-van Leeuwen Y, Berger MY, 2017. Dientamoeba fragilis colonization is not associated with gastrointestinal symptoms in children at primary care level. Fam Pract 34: 25-29.

13. Nagata N, Marriott D, Harkness J, Ellis JT, Stark D, 2012. Current treatment options for Dientamoeba fragilis infections. Int $J$ Parasitol Drugs Drug Resist 2: 204-215.

14. Garcia LS, 2016. Dientamoeba fragilis, one of the neglected intestinal protozoa. J Clin Microbiol 54: 2243-2250.

15. Engsbro AL, Stensvold CR, Nielsen HV, BytzerP, 2012. Treatment of Dientamoeba fragilis in patients with irritable bowel syndrome. Am J Trop Med Hyg 87: 1046-1052. 